\title{
Scalar field entanglement entropy for small Schwarzschild black hole
}

\author{
M V Teslyk and O M Teslyk \\ Taras Shevchenko Kyiv University, Physics Department, 2 Glushkov Prosp., Build. 1, \\ 03680 Kyiv, Ukraine \\ E-mail: machur@ukr.net
}

\begin{abstract}
We consider scalar field entanglement entropy generated with black hole of (sub)planck mass scale thus implying the unitary evolution of gravity. The dependence on the dimension of the Hilbert space for degrees of freedom located behind the horizon is taken into account. The obtained results contain polylogarithmic terms.

PACS numbers: 04.60.-m, 04.62.+v, 04.70.-s, 04.70.Dy
\end{abstract}

Submitted to: Class. Quantum Grav. 


\section{Introduction}

Bekenstein demonstrated in 1973 [1] that black hole $(\mathrm{BH})$ entropy $S_{\mathrm{BH}}$ is proportional to its horizon area $A$. As it is well-known from the 'no-hair' theorem the observer can determine the mass, angular momentum and the charge of the BH but no other properties thus giving rise to the entropy origin problem. The second problem is the information loss problem initiated by Hawking in 1975 [2] that in combination with [3] determined that the $\mathrm{BH}$ entropy $S_{\mathrm{BH}}=A / 4$ in Planck units. $\mathrm{BH}$ appeared to generate a large amount of entropy with no chance to read out any information almost about the matter falling below its horizon thus implying the non-unitary evolution. To date solution for the both problems is a challenging task for any theory of quantum gravity. At present many approaches to answer them have been proposed.

In 1996 Strominger and Vafa [4] proposed to consider BH entropy with the help of string theory. Such an approach has appeared to be a fruitful one; for more information on the topic one can read reviews such as [5] or the recent ones [6, 7] and references therein.

Another approach to the problems is based on the loop quantum gravity. It provides counting of microscopic BH microstates and therefore determinates its entropy. One can read more on the topic in [8, 9, 10] and in recent papers such as [11, 12]. In [13] authors applying the similar approach conclude that $\mathrm{BH}$ radiation spectrum should become less entropic as it evaporates. It leads to the possibility of information recovery from the $\mathrm{BH}$ due to the increasing role of quantum effects in the evolution of small BH. Such a conclusion witnesses in favor of information preservation with $\mathrm{BH}$ that was reconsidered first by Hawking in 2005 [14] and may interfere with our own results presented in [15].

Estimation of $\mathrm{BH}$ entropy with the help of quantum tunneling approach is proposed in such papers as [16.

In spite of variety of methods to calculate $S_{\mathrm{BH}}$ it is widely accepted that the entropy should be generated with the $\mathrm{BH}$ event horizon. As the horizon separates the whole spacetime into accessible and non-observable regions any distant observer should trace out all the degrees of freedom localized inside the BH. Therefore the horizon can serve not only as an entropy generator, but as a depository for the degrees of freedom giving rise to it. Such an approach was proposed with Srednicki [17]; for the review see [18]. Investigation of the $\mathrm{BH}$ horizon as the depository results in the holographic principle [19]; among all the publications on the topic we would like to mention [20, 21] where the authors use holography to calculate the entanglement entropy from AdS/CFT.

In [22] energy and entropy divergences arising in 't Hooft's brick wall model [23] are considered in the framework of the uncertainty principle. The authors raise the question of similarity between the entanglement and the statistical definitions of $\mathrm{BH}$ entropy. In [24] higher order corrections within the brick wall formalism for arbitrary spin have been found.

As the horizon separates the space into observable and non-observable one, it is logical to assume that $S_{\mathrm{BH}}$ originates from entanglement. Such a viewpoint is interesting 
since then there is no information loss at all and gravity obeys unitary evolution [14]. For the other approaches resulting in the unitary nature of the $\mathrm{BH}$ evolution one can read [25] and recent review [26]. The situation has much in common with the restricted access to some code represented in the Schmidt basis: being able to read some part of the code only, one concludes in non-zeroth entropy. But reducing the inaccessible part of the code reduces the entropy; the similar behavior should be observed for the decreasing horizon area. Such a process (i.e. accessing the part of the code only) can not be viewed as the one that generates entropy.

In this manuscript we apply the approach presented in [15] to BH of (sub)planck mass scale. We count the entanglement entropy of scalar field separated with the BH's horizon into two parts and compare it to $S_{\mathrm{BH}}$. We realize that the case of the planck scale BH is speculative a bit. As it is mentioned in [2] such small BHs can not be considered as some classical background metric for any quantum field. In such a case, one is expected to apply quantum gravity. But, as such a theory has not been built till now we try to take a look beyond.

On author's opinion, the (sub)planck mass scale BHs seem to be of great interest in the light of modern heavy ion collision experiments. In case of appearance during the LHC experiments small BHs will witness other dimensions via their spectrum radiation characteristics, and therefore we hope our investigation might be useful in the sphere. Also the results we derived may be helpful in the analysis of quark-gluon bag models.

Our approach has much in common with the others presented in [27, 28, 29]. However our approach has some differences. We estimate entropy via volume of $\mathrm{BH}$ and angular momentum while in [27, 28] shell volume near the horizon and momentum are utilized. As for [29] it is based on the thermal atmosphere surrounding the horizon.

This paper is organized as follows. In section 2 we present general idea of our model. Section 3 discusses the model itself. Entropy estimation and its analysis are presented in section 4. Discussion and conclusions one can read in section 5.

\section{Basic concepts}

Throughout the paper Planck units were used.

We consider Schwarzschild black hole of mass $M$ and consequently of radius $r=2 M$ and some scalar field surrounding it. The field is supposed to be in some pure state $|\Phi\rangle$ in the Kruskal frame of reference (FR) and to have no influence on the background metric (quasiclassical approach). Such a condition implies that the field energy is negligible comparatively to BH's mass.

In the following one should keep in mind that vacuum concept is not invariant with respect to FR, as it was shown by Unruh [30]. Hence, observers in different FRs will detect different states of the scalar field; this is the crucial point in this paper.

Observer in Kruskal FR will not detect the BH's event horizon. At the same time, observer from the accelerated, i.e. from the Schwarzschild, FR will detect the horizon with the particles being radiated with it. The creation and annihilation boson operators 
in both FRs are connected via the Bogoljubov transformations [2, 30]

$$
a^{\dagger}=\frac{1}{\sqrt{1-\zeta^{2}}} b_{\mathrm{out}}^{\dagger}-\frac{\zeta}{\sqrt{1-\zeta^{2}}} b_{\mathrm{in}}, \quad a=\frac{1}{\sqrt{1-\zeta^{2}}} b_{\mathrm{out}}-\frac{\zeta}{\sqrt{1-\zeta^{2}}} b_{\mathrm{in}}^{\dagger},
$$

where $a, a^{\dagger}$ are the annihilation and creation operators in the Kruskal FR, $b_{\text {in(out) }}, b_{\text {in(out) }}^{\dagger}$ are the annihilation and creation operators in the accelerated FR inside (outside) the horizon, and $\zeta$ is defined as

$$
\zeta=\exp (-4 \pi M \omega),
$$

where $\omega$ is the energy of the field quanta generated at the BH's event horizon under the Unruh effect.

The Kruskal field $|\Phi\rangle$ will be detected with the observer from the accelerated FR in the state

$$
|\Phi\rangle=\sqrt{\frac{1-\zeta^{2}}{1-\zeta^{2 N}}} \sum_{n=0}^{N-1} \zeta^{n}|n\rangle_{\text {in }}|n\rangle_{\text {out }}, \quad a|\Phi\rangle=0,
$$

where $N=N_{\text {in (out) }}$ is the dimension of the in- (out-) Hilbert subspaces [30]. Here the in- and out-components (denoted by the corresponding subscripts) describe the parts of the field under and above the horizon. We emphasize that now we are working with a single mode of the scalar field only. Later we will integrate over all $\omega$ possible to take into account all the modes.

As one can see, (21) is exactly the Schmidt decomposition [31, 32], and hence one obtains density matrices of the in- and out-components

$$
\begin{aligned}
& \rho_{\text {in }}=\operatorname{Tr}_{\text {out }}|\Phi\rangle\left\langle\Phi\left|=\frac{1-\zeta^{2}}{1-\zeta^{2 N}} \sum_{n=0}^{N-1} \zeta^{2 n}\right| n\right\rangle_{\text {in }}\langle n|, \\
& \rho_{\text {out }}=\operatorname{Tr}_{\text {in }}|\Phi\rangle\left\langle\Phi\left|=\frac{1-\zeta^{2}}{1-\zeta^{2 N}} \sum_{n=0}^{N-1} \zeta^{2 n}\right| n\right\rangle_{\text {out }}\langle n| .
\end{aligned}
$$

As we see, different observers handle different density matrices. Though the Kruskal observer detects the pure state $|\Phi\rangle$, the accelerated one, because of having access to the out-component of the field (i.e. to the outgoing radiation) only, detects a mixture with entropy

$$
\begin{aligned}
\sigma(N, \zeta) & =-\operatorname{Tr} \rho_{\text {out }} \ln \rho_{\text {out }}=-\frac{1-\zeta^{2}}{1-\zeta^{2 N}} \sum_{n=0}^{N-1} \zeta^{2 n} \ln \left(\frac{1-\zeta^{2}}{1-\zeta^{2 N}} \zeta^{2 n}\right) \\
& =-\ln \frac{1-\zeta^{2}}{1-\zeta^{2 N}}-\left(\frac{\zeta^{2}}{1-\zeta^{2}}-N \frac{\zeta^{2 N}}{1-\zeta^{2 N}}\right) \ln \zeta^{2}
\end{aligned}
$$

where the following relation

$$
\sum_{n=0}^{N-1} n \zeta^{2 n}=\left.\frac{1}{2 \ln \zeta} \partial_{\alpha} \sum_{n=0}^{N-1} \zeta^{2 n \alpha}\right|_{\alpha=1}=\frac{\left(1-\zeta^{2 N}\right) \zeta^{2}-N\left(1-\zeta^{2}\right) \zeta^{2 N}}{\left(1-\zeta^{2}\right)^{2}}
$$

has been used. 
As one can see from (3),$\sigma(N, \zeta)$ depends on 2 parameters: $N$ and $\zeta$. The main problem here is to estimate the value of $N$. It is easy in the asymptotic of large $\mathrm{BH}$, as one can use the limit $N \rightarrow \infty$ then; such an asymptotic is popular in the literature. In [15] it was applied too. However, here we consider small BHs with mass $M \leq 1$ and therefore have to take into account finiteness of $N$. Direct estimation of the magnitude of $N$ may be done via calculation of the field energy. However it leads to the integral which can be solved approximately only and therefore is not discussed here. Anyway we expect $N \gg 1$ since otherwise the scalar field will influence the $\mathrm{BH}$ and thus violate the quasiclassical approach. Such an assumption seems to be reasonable and to have no contradictions with the model. For BH with (sub)planck mass $M \leq 1$ and for the small rest mass of scalar field quanta $N$ must be large enough to encode all the degrees of freedom.

\section{Model construction}

Expression (3) is written for some mode with fixed parameters of the radiated field component. Model construction requires correct contribution estimation of all the modes to the entropy, that is of the system symmetry and of the energy spectrum determined with $\omega$.

Due to the spherical symmetry we must take into account the contributions from all the angular momenta $l$ and its projections $-l \leq \mu \leq l$ possible. The range on $l$ is well-defined and can be written in the following form:

$$
0 \leq \sqrt{l(l+1)} \leq \sqrt{L(L+1)}=r p=2 M \sqrt{\omega^{2}-m^{2}},
$$

where $p$ is the momentum of the field quantum radiated away.

In such a case the entropy from (3) should be multiplied with

$$
\sum_{l=0}^{l=L} \sum_{\mu=-l}^{\mu=l} 1=4 M^{2}\left(\omega^{2}-m^{2}\right)+\frac{\sqrt{16 M^{2}\left(\omega^{2}-m^{2}\right)+1}+1}{2} .
$$

But, taking into account angular degrees of freedom is not enough. In (3) $\sigma(N, \zeta)$ is defined for the fixed $\omega$ only. As we want to estimate the contribution from all the modes we should integrate over all the $\omega$ possible. Therefore we write down the following integral for the radiation entropy $S(N, M, m)$ :

$$
S(N, M, m)=\frac{V}{(2 \pi)^{3}} \int_{m}^{M} \sum_{l=0}^{l=L} \sum_{\mu=-l}^{\mu=l} \sigma(N, \zeta) \mathrm{d} \omega,
$$

where $V=4 \pi r^{3} / 3=2^{5} \pi M^{3} / 3$ is the $\mathrm{BH}$ volume confined with the horizon and $m$ is the rest mass of the radiated quanta. The upper integral bound is equal to $M$ here because the energy of the field quanta can not exceed BH mass. Substituting (3) and (4) into the integral we obtain for the radiation entropy

$\frac{S(N, M, m)}{S_{\mathrm{BH}}}=\frac{M}{6 \pi^{3}} \int_{m}^{M} \sigma(N, \zeta)\left[1+8 M^{2}\left(\omega^{2}-m^{2}\right)+\sqrt{1+16 M^{2}\left(\omega^{2}-m^{2}\right)}\right] \mathrm{d} \omega,(5)$

where $S_{\mathrm{BH}}=4 \pi M^{2}$ is the Bekenstein-Hawking entropy and $\zeta$ is defined in (1). 


\section{Entropy estimation}

Before we proceed, let us make some estimations of $m$. In case of being not equal to 0 $m$ should be of elementary particles mass order, i.e.

$$
m=0 \quad \text { or } \quad 10^{-23} \leq m \leq 10^{-18} \quad \Rightarrow \quad m \gtrsim 0,
$$

where $10^{-23}$ is of order of the electron mass $m_{e}$ and $10^{-18}$ is of order of the $\mathrm{Z}^{0}$ boson mass $m_{Z^{0}}$. So it should be taken into account that $m$ is a small number.

The integral (5) can not be calculated directly due to the strong integrand dependence on the integral boundaries. The situation is complicated with the exact entropy dependence on $N$. In [15] the dependence on $N$ had been neglected because of the large BH mass, but here we can not do the same trick.

To estimate the entropy at first we decompose $\sigma(N, \zeta)$ from (3) into series

$$
\sigma(N, \zeta)=\sum_{n=1}^{N} \zeta^{2 n}\left(\frac{1}{n}-2 \ln \zeta\right)-N \zeta^{2 N}\left(\frac{1}{N}-2 \ln \zeta\right)+\mathcal{O}\left[\zeta^{2(N+1)}\right] .
$$

Due to (1) $\zeta$ exponentially depends on $\omega$, and thus such a decomposition is good at the higher integral bound $\omega=M$. But one can argue that at the lower integral bound $\omega=m$ such a series expansion may fail: due to smallness of $m$, that follows from (6), $\zeta$ will not differ from unity a lot. However the neglected terms in the expansion are of the order $\zeta^{2(N+1)}$, so here we must take into account not the mass $m$ itself but the product $N m$ in the exponent. As we discussed at the end of Section 2 the number $N$ is expected to be large since otherwise the scalar field will influence the $\mathrm{BH}$ thus violating the quasiclassical approach. So we conclude that the decomposition (7) is applicable at the whole range $m \leq \omega \leq M$ but except the case $m=0$.

The second step is decomposition of the square root term in the integrand from (5) . Expanding it into series with respect to $\omega$ one meets the problem at the upper bound of the integral since $M \leq 1$, so we use the following trick. As

$\forall \omega \in[m, M] \quad 1+16 M^{2} \omega^{2}>16 M^{2} m^{2} \quad \Rightarrow \quad(1+4 M \omega)^{2}>8 M \omega+16 M^{2} m^{2}$,

that allows to rewrite the square root from (5) in the following form

$$
\begin{aligned}
\sqrt{1+16 M^{2}\left(\omega^{2}-m^{2}\right)} & =(1+4 M \omega) \sqrt{1-\frac{8 M \omega+16 M^{2} m^{2}}{(1+4 M \omega)^{2}}} \\
& =(1+4 M \omega)\left\{1-\frac{4 M \omega+8 M^{2} m^{2}}{(1+4 M \omega)^{2}}+\mathcal{O}\left[\frac{\left(M \omega+2 M m^{2}\right)^{2}}{(1+4 M \omega)^{4}}\right]\right\} \\
& \approx 4 M \omega+\frac{1-8 M^{2} m^{2}}{1+4 M \omega} .
\end{aligned}
$$

Estimating the error for (8) one can notice that the expression in the square brackets increases with $\omega$ decreasing. As a result the error of the decomposition applied will be of order $\mathcal{O}\left[M^{2} m^{2}(1+2 m)^{2}(1+4 M m)^{-4}\right]$ and thus is small due to (6) . 
Substituting (7) and (8) to (5) we obtain

$$
\frac{S(N, M, m)}{S_{\mathrm{BH}}} \approx-\left.\frac{1}{24 \pi^{3}}\left(\sum_{n=1}^{N} \alpha_{n}-N \alpha_{N}\right)\right|_{\omega=m} ^{\omega=M},
$$

where

$$
\begin{aligned}
\alpha_{n}= & \zeta^{2 n}\left[\frac{1+4 M \omega+8 M^{2}\left(2 \omega^{2}-m^{2}\right)+32 M^{3} \omega\left(\omega^{2}-m^{2}\right)}{n}\right. \\
& \left.+\frac{1+6 M \omega+8 M^{2}\left(2 \omega^{2}-m^{2}\right)}{\pi n^{2}}+\frac{3+16 M \omega}{4 \pi^{2} n^{3}}+\frac{1}{2 \pi^{3} n^{4}}\right] \\
& +\left(1-8 M^{2} m^{2}\right)(2 \pi-1 / n) \mathrm{e}^{2 \pi n} \operatorname{Ei}[-2 \pi n(1+4 M \omega)],
\end{aligned}
$$

where $\operatorname{Ei}(x)=\int_{-\infty}^{x} \mathrm{e}^{-t} / t \mathrm{~d} t$.

Expressions (9) and (10) give an approximate estimation for the scalar field entanglement entropy for small $\mathrm{BH}$.

From (9) it is hard to achieve the power law since in any order the corresponding terms will vanish after substituting the integral boundaries. We have no explanation of this fact but except that for the $\mathrm{BH}$ of the (sub)planck mass scale the radiation spectrum should change to take into account quantum gravity effects. Quite similar conclusion was made in [13] with the help of loop quantum gravity also.

The term proportional to logarithm of $S_{\mathrm{BH}}$ may be obtained in the following way. As one can see, the leading-order term from (101) is proportional to $\zeta^{2 n} / n$. Neglecting with the higher powers of $n$ we obtain from (91) and (10) that

$$
\frac{S(N, M, m)}{S_{\mathrm{BH}}} \propto-\left.\left(\sum_{n=1}^{N} \frac{\zeta^{2 n}}{n}-\zeta^{2 N}\right)\right|_{\omega=m} ^{\omega=M},
$$

that after setting $N \rightarrow \infty$ and applying (1) transforms to

$$
\frac{S(\infty, M, m)}{S_{\mathrm{BH}}} \propto \ln \frac{1-e^{-8 \pi M^{2}}}{1-e^{-8 \pi M m}} .
$$

Taking into account (6) one can notice that $M m \ll 1$ and then easily extract the term proportional to $\ln M$ from (11). However one should keep in mind that the entanglement entropy is expressed in the units $S_{\mathrm{BH}}$ already. So the derived term is not the logarithm correction in its common sense, but the one proportional to it.

As we see from (9) the terms containing higher powers of $1 / n$ transform to polylogarithms of order 2,3 and 4 . So we have obtained the other corrections to the scalar entanglement entropy.

Finally we would like to give an upper bound for the scalar entanglement entropy. As one can notice from (5) the integrand is non-negative for any values of $\omega$ and $N$. Therefore from (9) it follows that the entropy takes maximum values at its boundary:

$$
\frac{S(N, M, m)}{S_{\mathrm{BH}}} \leq \frac{S(N \rightarrow \infty, 1,0)}{4 \pi} \approx 1.462 \cdot 10^{-3} .
$$

Therefore the scalar field entanglement entropy can not be responsible for all the entropy generation: its contribution is less than $1 \%$. 


\section{Discussion}

Summing up we estimated the radiation entropy of the scalar field generated with the horizon of $\mathrm{BH}$ with mass $M \leq 1$. This paper complements [15] where the case $M>1$ was considered. It is based on the similar principles.

In the paper we considered the influence of the dimension number $N$ of the in(out)side Hilbert subspace with respect to the BH horizon. $N$ is usually taken to be infinite for simplicity, but here we could not do so due to the smallness of $\mathrm{BH}$. Estimation of the magnitude of $N$ deserves further research.

The results demonstrate no area law dependence. We suppose this as a consequence of quantum gravity effects. The term proportional to logarithm of $\mathrm{BH}$ area is obtained. It is not the logarithm correction term in the common sense: it is the product of logarithm and $S_{\mathrm{BH}}$ itself. The other correction terms contain polylogarithms of order from 2 to 4 .

The upper bound of the scalar entanglement entropy does not exceed $1 \%$ of $S_{\mathrm{BH}}$. Comparing the entanglement entropy upper bound to [15] we notice that its contribution is almost the same. Such a result follows from the fact that for the upper bound estimation on the entropy the similar assumptions were used (i.e. $N \rightarrow \infty$ ). Taking the finiteness of $N$ will reduce the scalar entropy contribution to $S_{\mathrm{BH}}$ even more but will not change the result significantly. So we conclude that the scalar field entanglement entropy does not dominate in $S_{\mathrm{BH}}$.

Smallness of the scalar field contribution to BH entropy might be a consequence of our restrictions to the scalar field only. BH entropy is detected via the particles radiated away. So the degrees of freedom encoded with other quantum numbers should play more significant role. Here the analogy with some register may be observed. One-symbol language provides linear growth of the number of possible states of the register, while even the binary language provides the exponential one. In such a case other quantum numbers such as spin and its projections are expected to increase the entanglement entropy contribution significantly enough. Such a supposition needs further investigation.

Contribution of the scalar entanglement entropy appeared to be small comparatively to the results obtained in [17, 18, 33]. Such a discrepancy should have been expected due to the differences in the models and assumptions considered.

Our approach has much in common with the one presented in [27, 28] where the upper bound on the entropy is derived. Compared to the papers here we present the analytical expression for the entropy for small $\mathrm{BH}$ taking into account its dependence on $N$.

As is well-known, some of the LHC detectors are designed to explore 'new physics'; it implies looking for the additional dimensions. In case there are such, the $\mathrm{BH}$ might appear during the collisions and therefore might be detected via its Hawking radiation. The paper may be helpful for searching for possible BH generation during collisions. Despite here we considered the case of 3+1-dimensional BH, which can not be observed 
on the LHC, the manuscript may shed some light on the topic since such BH is expected to be small: its mass can not exceed 1 due to the energy restrictions. Also the presented results can be helpful for the analysis of quark-gluon bag model or similar ones.

\section{Acknowledgments}

We are thankful to Belokolos E D for all the valuable discussions and remarks during preparation of this work. 
[1] Bekenstein J D. Black hole and entropy. Phys. Rev. D, 7:2333-46, 1973.

[2] Hawking S W. Particle creation by black holes. Commun. Math. Phys., 43:199-220, 1975.

[3] Carter B Bardeen N D and Hawking S W. The four laws of black hole mechanics. Commun. Math. Phys., 31:161-70, 1973.

[4] Strominger A and Vafa C. Microscopic origin of the bekenstein-hawking entropy. Phys. Rev. B, 379:99-104, 1996.

[5] Horowitz G T. Black holes, entropy, and information.

[6] Prester P D. $\alpha^{\prime}$-corrections and heterotic black holes. 2010.

[7] Gomes J. Quantum entropy of supesymmetric black holes. 2011.

[8] Khriplovich I B. Holographic bound and spectrum of quantized black hole. page 5, 2004.

[9] Khriplovich I B. Quantized black holes, their spectrum and radiation. Phys. Atom. Nucl., 71:671$80,2005$.

[10] Jacobson T. Renormalization and black hole entropy in loop quantum gravity. Class. Quantum Grav., 24:4875-79, 2007.

[11] Bianchi E. Black hole entropy, loop gravity, and polymer physics. Class. Quantum Grav., 28:114006, 2011.

[12] Livine E R and Terno D R. Entropy in the classical and quantum polymer black hole models. Class. Quantum Grav., 29:224012, 2012.

[13] Silva C A S and Landim R R. A note on black hole entropy, area spectrum, and evaporation. page 4, 2010.

[14] Hawking S W. Information loss in black holes. Phys. Rev. D, 72:084013, 2005.

[15] Belokolos E D and Teslyk M V. Scalar field entanglement entropy of a schwarzschild black hole from the schmidt decomposition viewpoint. Class. Quantum Grav., 26:235008, 2009.

[16] Ran Li. Logarithmic entropy of black hole in gravity with conformal anomaly from quantum tunneling approach. Europhys. Lett., 96:60014, 2011.

[17] Srednicki M. Entropy and area. Phys. Rev. Lett., 71:666-9, 1993.

[18] Shankaranarayanan S Das S and Sur S. Black hole entropy from entanglement: a review. 2008.

[19] Susskind L. The world as a hologram. J. Math. Phys., 36:6377-96, 1995.

[20] Ryu S and Takayanagi T. Holographic derivation of entanglement entropy from ads/cft. Phys. Rev. Lett., 96:181602, 2006.

[21] Ryu S and Takayanagi T. Aspects of holographic entanglement entropy. J. High Energy Phys., 0608:045, 2006.

[22] Brustein R and Kupferman J. Black hole entropy divergence and the uncertainty principle. Phys. Rev. D, 83:124014, 2011.

[23] G 't Hooft. On the quantum stricture of a black hole. Nucl. Phys. B, 256:727-45, 1985.

[24] Wontae K and Shailesh K. Higher order wkb corrections to black hole entropy in brick wall formalism. 2012.

[25] You L Zhang B, Cai Q and Zhan M. Hidden messenger revealed in hawking radiation: a resolution to the paradox of black hole information loss. Phys. Lett. B, 675:98-101, 2009.

[26] Solodukhin S N. Entanglement entropy of black holes. Living Rev. Relativity, 14(8), 2011.

[27] Lambiase G Iorio A and Vitiello G. Quantization of scalar fields in curved background, deformed hopf algebra and entanglement. 2002.

[28] Lambiase G Iorio A and Vitiello G. Etangled quantum fields near the event horizon and entropy. Ann. Phys., 309:151-65, 2004.

[29] Jacobson T and Paretani R. Black hole entanglement entropy regularized in a freely falling frame. Phys. Rev. D, 76:024006, 2007.

[30] Unruh W G. Notes on black-hole evaporation. Phys. Rev. D, 14:870-892, 1976.

[31] Schmidt E. Zur theorie der linearen und nichlinearen intergralgleihungen. Math. Ann., 63:433, 1907.

[32] Ekert A and Knight P L. Entangled quantum system and the schmidt decomposition. American Journal of Physics, 63:415, 1995. 
[33] Seriu M Mukohyama S and Kodama H. Thermodynamics of entanglement in schwarzchild spacetime. Phys. Rev. D, 58:064001, 1998. 